\title{
Contactless pressing of a sheet
}

\author{
Karel Adámek ${ }^{1, \text { a }}$, Josef Žák ${ }^{1}$, Pavel Kavan ${ }^{1}$, Pavel Šidlof ${ }^{1}$, Jan Kolář ${ }^{1}$ \\ ${ }^{1}$ VÚTS Liberec, a.s., 46001 Liberec XI, Czech Republic
}

\begin{abstract}
The paper summarizes the results of several numerical flow simulations of force influence of air flows on the treated paper sheet to get a contactless fixing of the treated material during its passage through the machine and to prevent the contact of the sheet with the structural elements in the surroundings.
\end{abstract}

\section{Subject and goal of the solution}

The sheet of printed paper is drawn through the system of cylinders on printing machine, fixed at its front edge. The problem is that in certain moments of the process the free rear end of the sheet is drawn away from the cylinder surface and it is possible to touch the neighbouring structure then. The result of it could be the damaged print (smudging) or damaged paper surface (abrasion).

The goal of the study is the definition of the reason of this phenomenon, its analysis and a design of possible improvement.

\section{Force effects}

The first part deals with various possible force effects on the treated sheet. As starting points there are the previous solutions [1], [2], [3] with a design of contactless pressure using aerodynamic forces. The solution is divided into two parts - elastic, where there are defined the forces needed for the deformation of the treated sheet into the cylindrical shape and aerodynamic, where the flows are modelled in such a manner to get force effects similar to the results of elasticity calculations.

\subsection{Flow field around the rotating cylinders}

Due to the complicated surroundings, the 2D modelling [4] was used, only, with a cylinder length of $774 \mathrm{~mm}$, a diameter of $460 \mathrm{~mm}$, a peripheral velocity of $3 \mathrm{~m} / \mathrm{s}$, and an angular velocity of $13 \mathrm{rad} / \mathrm{s}$, respectively. It is possible to state the hypothesis that the cylinder faces have no significant influence on the image of the flow field in the middle part of cylinders, so the results of $2 \mathrm{D}$ modelling are quite exact. The main results are presented below. At all illustrations of flow fields the maximum value is red and the minimum one is blue. Absolute values are not important here; more important is the character or tendency of observed quantity.

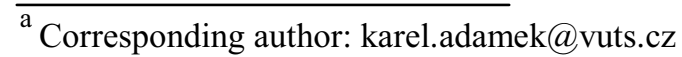

\subsubsection{Pressure cylinder with a fixing pin}

The results of modelling for basic revolutions $(3 \mathrm{~m} / \mathrm{s}$ or $13 \mathrm{rad} / \mathrm{s})$ are as follows:

Velocity field (figure 2.1-1) - the air volume in the wedge and the boundary layer at the cylinder surface are rotating together with the cylinder, the velocity is decreasing with an increased diameter.

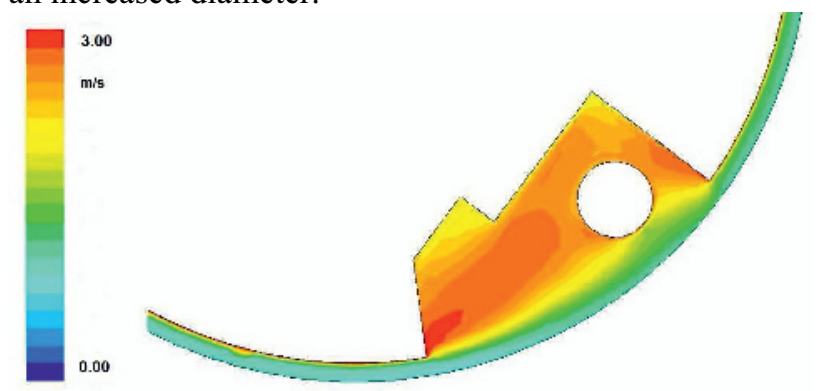

Figure 2.1-1. Velocity field.

Static pressure (figure 2.1-2) - the local pick of $1,5 \mathrm{~Pa}$ is situated at the leading edge, followed by a small under pressure of some $0,1 \mathrm{~Pa}$ along the perimeter of the cylinder.

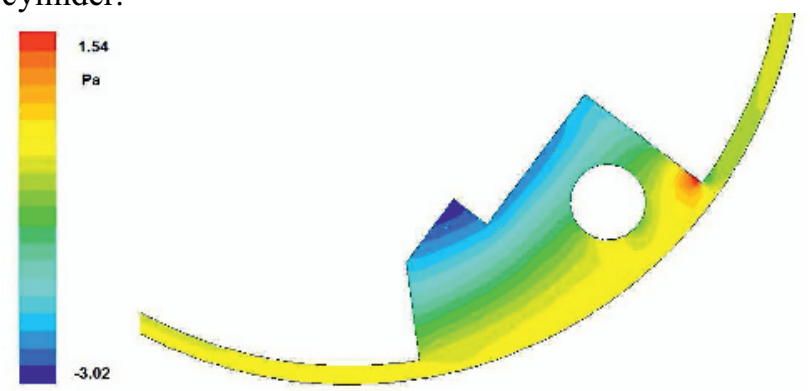

Figure 2.1-2. Pressure field. 


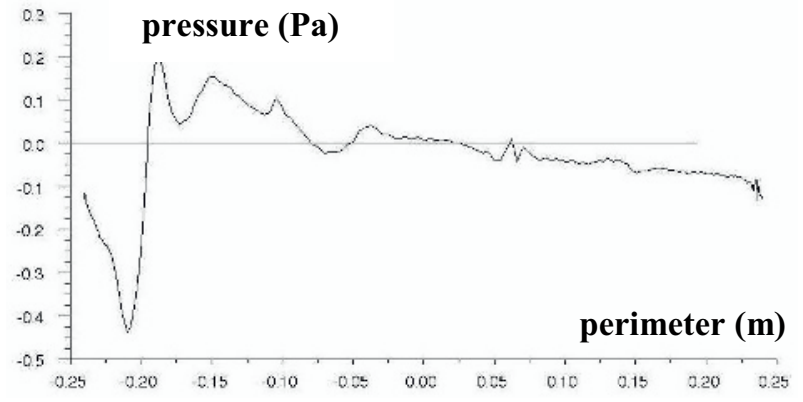

Figure 2.1-3. Pressure profile along the rotating surface with pin.

The graph of static pressure along the perimeter for the same case (figure 2.1-3) - the local pick is situated in the wedge. The graph presents one half of perimeter - two sheets on the cylinder perimeter.

From a record of several positions during the cylinder rotation it is clear that the pressure field in the surrounding of the rotating cylinder is practically undisturbed.

\subsubsection{Model of the cylinder system}

The next model simulates the system of three cylinders (transmitting, pressing and printing), used on printing machine. They are alternatively furnished by covers and the periodical boundary condition is defined in the direction of the sheet movement so that it is simulated the influence of the neighbouring systems, too. (Remark: the position of the printing cylinder is not right - mistake but on the flow field between the pressing and transmitting cylinders it has not any basic influence.) The transmitting cylinder is solved as a model of moving mesh; the next cylinders (pressing and printing) are solved as smooth surfaces with a defined peripheral velocity. The pressing cylinder is modelled here without wedges - see above.

From the global unsteady solution it is here presented a complicated flow field for one static position, only, both with mounted covers - see figure 2.1-4 to figure 2.1-6 and without covers - see figure 2.1-7 to figure 2.1-9. We can see that the results without covers are similar, only the pressure values are "shifted" a little up.
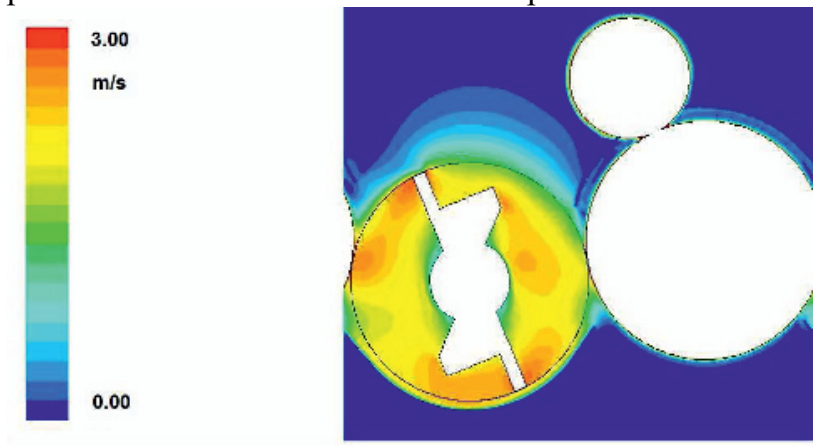

Figure 2.1-4. Velocity field - with covers.
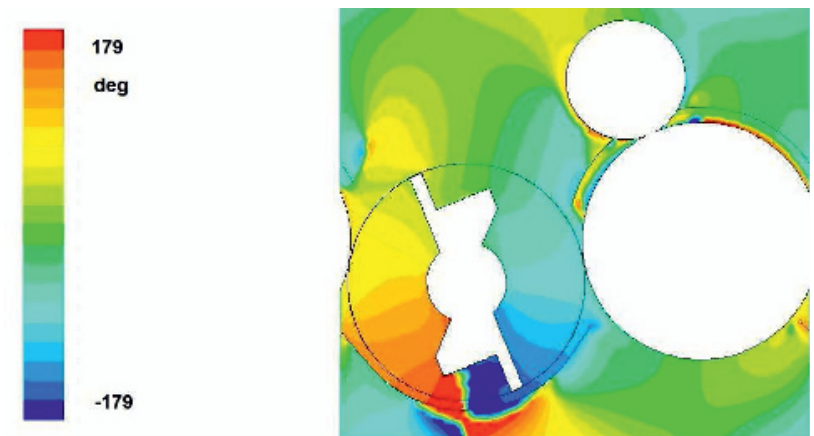

Figure 2.1-5. Directional field (direction from $0^{\circ}$ to $180^{\circ}$ ) with covers.

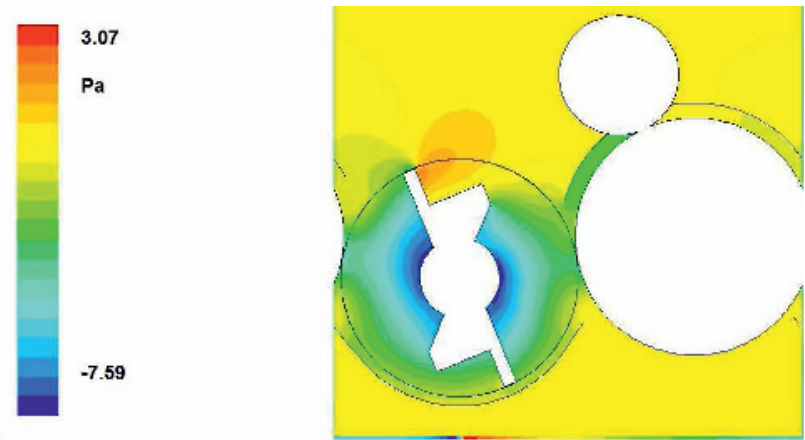

Figure 2.1-6. Pressure field - with covers.
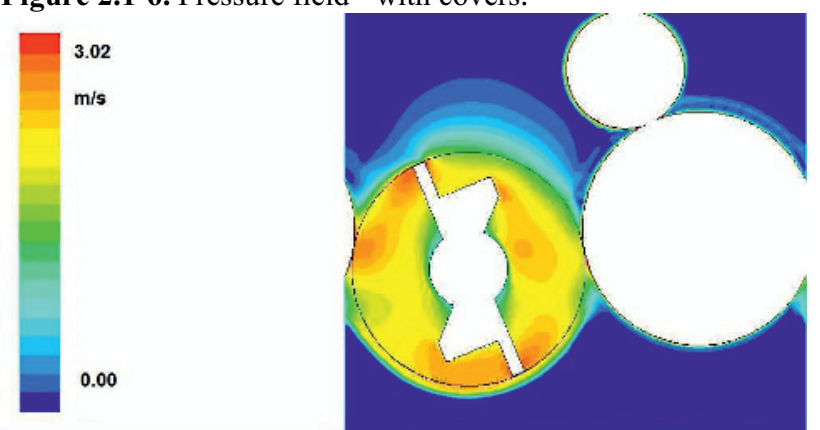

Figure 2.1-7. Velocity field - without covers.
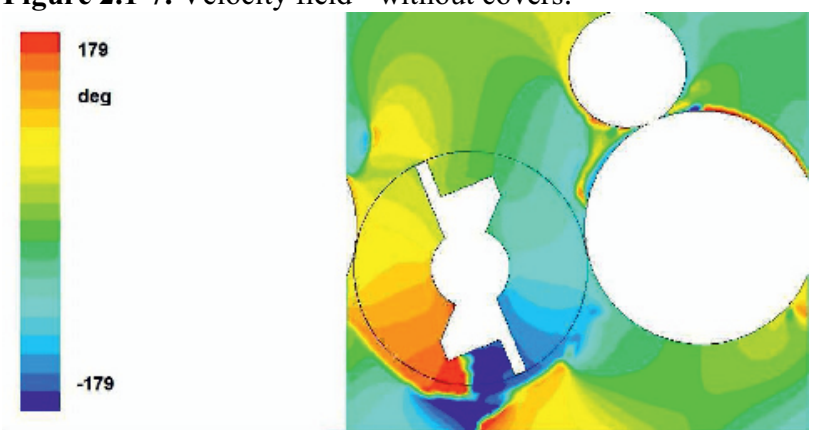

Figure 2.1-8. Directional field (direction from $0^{\circ}$ to $180^{\circ}$ ) without covers.
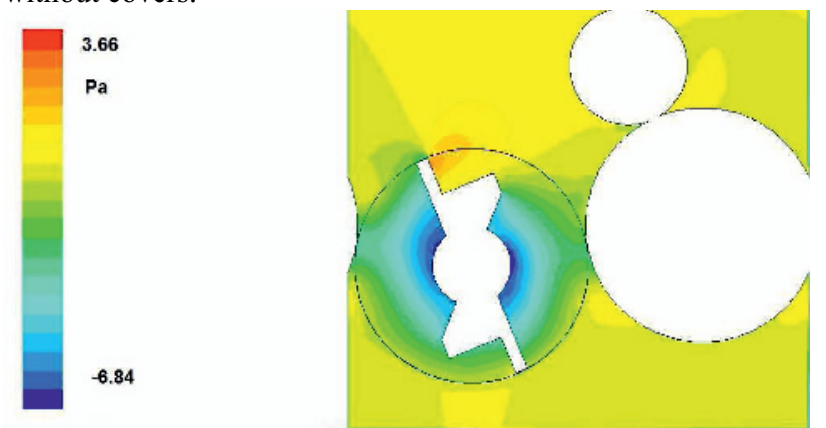

Figure 2.1-9. Pressure field - without covers. 


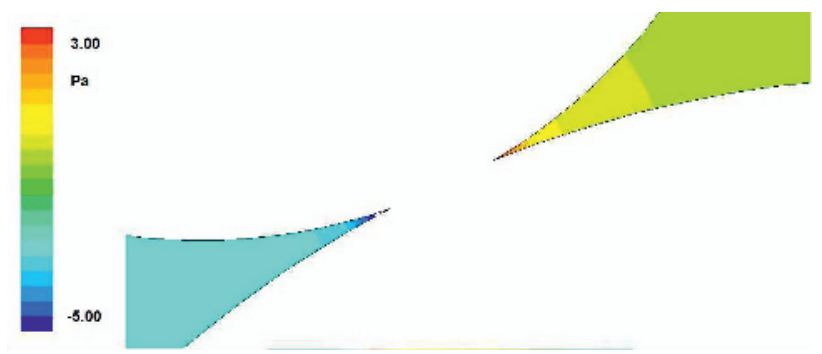

Figure 2.1-10. Detail of the pressure field in the vicinity of the contact point (rotation direction $\swarrow$ )

figure 2.1-10 shows the detail of the pressure field in the vicinity of the contact point of two parallel rotating cylinders in contact. The friction of the rotating surfaces of the pressing and printing cylinders in the viscous air induces the complicated air flow in the surroundings which is pressed into the wedge-shaped space and then it is flowing back in the surroundings. The arising secondary flow could have sometimes an adverse influence on the treated material. In general, the overpressure is observable at the inlet side where both the rotating surfaces are coming near. And vice versa, at the outlet side, where the rotating surfaces are taking away, it is arising the under pressure, filled by the air flow from the surroundings. For a more detailed analysis and application see for instance [5], [6].

\subsection{Mass forces}

Centrifugal force, acting on mass element $d m$ on the cylinder radius $R$ rotating with angular velocity $\omega$ is

$$
d O=d m \cdot R \cdot \omega^{2}
$$

The force is acting always in the radius direction, so in the fix coordinate system its direction is changing with the cylinder rotation. In every moment it removes the sheet from the cylinder surface.

The next mass force is the own weight

$$
d G=d m \cdot g
$$

Its direction is always ,down“, also in the upper part of the cylinder the sheet is pressed to the cylinder surface and in the lower part it is pushed away. This change is harmonic in time. The radial component $d G r$ will slightly modulate the value of centrifugal force $d O$. For the given dimensions, such modulation is not essential. The effect of the tangential component $d G t$ is alternate during each turn so that the treated sheet on the cylinder surface is stretched or pressed.

Checking calculation of ,centrifugal pressure“, it means of the continual centrifugal load, acting on the sheet rotating together with the cylinder, after

$$
p=m \cdot r \cdot \omega^{2} \text { or } p=m \cdot v^{2} / r,
$$

gives for a light paper of $80 \mathrm{~g} \cdot \mathrm{m}^{-2}$ and a basic angular velocity of $13 \mathrm{rad} / \mathrm{s}$ the value of $3,1 \mathrm{~Pa}$. Comparing with figure $2.1-2$ it is similar to the result of simulated aerodynamic pressure in the vicinity of the rotating cylinder.

For heavier sheets, the effect of centrifugal force is higher, therefore, the machine is presently equipped by various mechanical supports, etc. Probably, the observed sheet fluttering during its passage through the machine is given preferably by mass forces and by the sheet bend stiffness and not by aerodynamic forces from the cylinder rotation.

Remark: The conclusion is identical with the conclusions of another publications, as for instance at the simulation of the rotating drum of carding machine [1], [2], [3] where the aerodynamic forces due to the rotation of the profiled (dented) drum surface can be neglected in comparison with another forces (mass, friction) during the process.

The above mentioned formula (3) shows that the increasing of the machine output (it means of the higher peripheral velocity of the sheet passage through the machine) by an increase of cylinder revolutions means the quadratic increasing of centrifugal force acting on the treated sheet. A better solution of the output increasing should be the diameter increasing at the same revolutions, where the centrifugal force is increasing in linear manner, only. But it is the problem of larger outline dimensions of the machine.

\subsection{Paper sheet deformation}

For next calculations, the following mechanical parameters of the treated sheet were defined from the result of a tensile strength test.

Table 1. Mechanical properties of paper sheet.

\begin{tabular}{|c|c|c|}
\hline Mass & Young modulus & Thickness \\
\hline $\mathrm{g}^{\cdot} \mathrm{m}^{-2}$ & $\mathrm{MPa}$ & $\mathrm{mm}$ \\
\hline 80 & 4650 & 0,10 \\
\hline 200 & 8700 & 0,20 \\
\hline
\end{tabular}

The large and thin sheet is very flexible. In general, it is known that for bend beams of such character they have usually a little higher bend stiffness in comparison with the usual forms of beam cross-section. Therefore, in formulas for bend beams it is used the term $E /\left(1-\mu^{2}\right)$ instead of Young modulus $E$, where the $\mu$ is the Poisson's ratio of cross contraction - for paper sheets it is not known.

The general equation of the deflexion line is

$$
\left(d^{2} w(x) / d x^{2}\right) /\left[1+(d w(x) / d x)^{\wedge} 2\right]^{\wedge}(3 / 2)=-M(x) /(E \cdot I)
$$

and due to a large deformation of thin sheets, it is not possible to neglect the term $d w(x) / d x) \wedge 2$ in the denominator, comparing to the 1 , as it is usually used for small deformations. For continual load, the Schwedler's formula is used as 


$$
d^{2} M(x) / d x^{2}=-q(x)
$$

and due to large deformations, this continual load should be written as

$$
q(x)=q / \cos \alpha(x)=q \cdot \sqrt{ }\left(1+(d w(x) / d x)^{\wedge} 2\right) .
$$

If the required form of the flexural line should be a circle

$$
w(x)^{2}+x^{2}=R^{2},
$$

the last unknown parameter is the continual load $q(x)$. One part of this load creates mass forces above, the rest should be created, for instance by a contactless aerodynamic effect of free flows.

The analytical solution can be approximated, only, because the exact one is known from the essential course of elasticity: The deflexion of a beam as with constant torsion $1 / R$ of deflexion line is possible to get only by a moment (by a pair of forces), deforming the beam. But in a contactless manner it is not possible to act with such a force. The conclusion was verified using an analytical solution Matlab [7] - the analytical continual load for the defined deflexion was not found.

The next solution [7] was realized using the method of finite elements (FEM). During the serial of linear calculations the contact of the sheet with the cylindrical surface was ,piloted“ by the changes of boundary conditions and the changes of air pressure in individual calculations The simple FEM model of the paper sheet was created from the mesh of 36 shell elements of 8 nodes, only, with a thickness of $0,2 \mathrm{~mm}$, in 6 rows and 6 columns. The solution procedure was as follows:

a) At the upper horizontal row of node points it was defined the fixing of the upper edge of paper sheet (zero shift, zero rotation).

b) The FEM model was loaded by some overpressure to get the contact of the second line of horizontal node points with the cylindrical surface. It was burdened with a certain pressure and its value has been changed gradually until there is a contact of the second horizontal range of nodal points with the cylindrical surface.

c) Using boundary conditions, there are defined constrained shiftings of this line of node points, to be situated just on the cylindrical surface and the pressure has been further changed.

By an analogous manner, i.e., by repeating points b) and c), the contact of the paper sheet with the cylindrical surface was reached.

As an illustration, figure 2.3-1 shows the result of one intermediate position of deformation: blue is the sheet part in contact, not blue is the necessary deformation up to reach the contact.

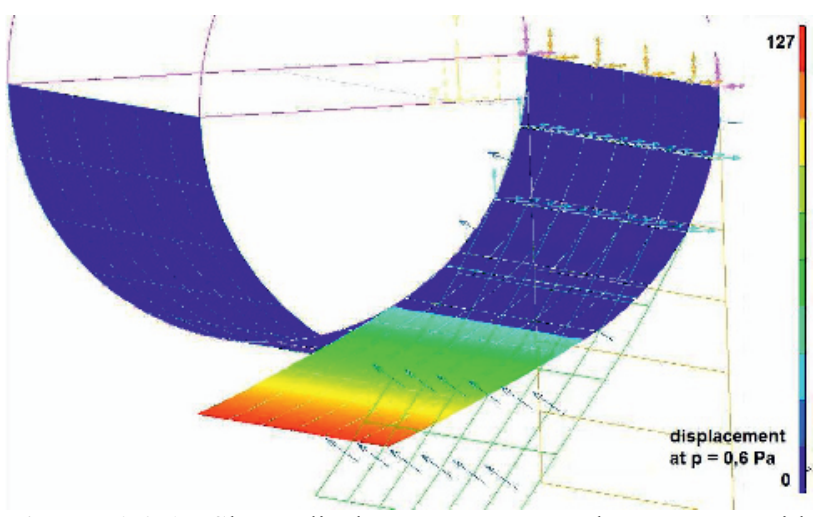

Figure 2.3-1. Sheet displacement up to the contact with cylindrical surface (some intermediate position)

The next graph in figure 2.3-2 shows the pressure increasing for holding the deformed sheet along the cylindrical surface - for the 1 st to the 6 th row of nodes.

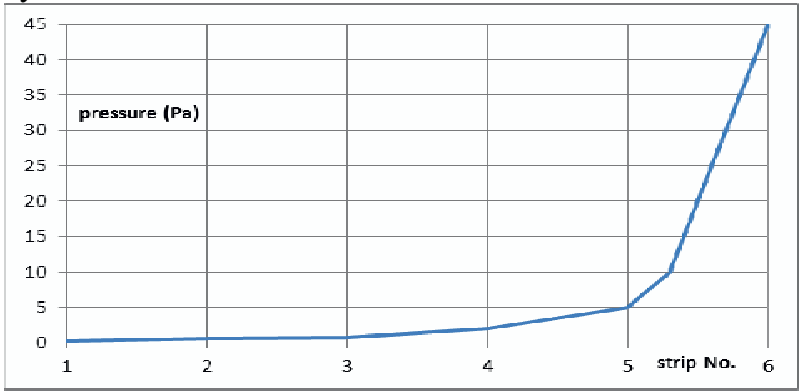

Figure 2.3-2. Pressure necessary for holding on the sheet along the cylindrical surface (1st to 6 th row of nodes)

From the summary of all the results it is clear that by pressing the upper horizontal edge of the paper sheet to the cylindrical surface, the bending moment is created so that the beginning bend of the given paper needs a minimum value of air pressure (of about $1 \mathrm{~Pa}$ ). With an increasing distance from the upper edge, the needed pressure value increases, too, gradually at $1-10 \mathrm{~Pa}$. To get the contact of the lower edge of the paper sheet with the cylindrical surface, the pressure of some tenths of $\mathrm{Pa}$ is needed.

Using the pressure of $0,6 \mathrm{~Pa}$, the contact of two first rows of node points is reached. Using the pressure of $0,8 \mathrm{~Pa}$ in the next phase, the contact is realized for the third and partially for the fourth row of node points.

The total air pressure, needed for the retaining of the paper sheet on the rotating cylindrical surface, can be defined approximately as the sum of the "over pressure" from mass forces and the pressure found out by this FEM method. For the paper of $200 \mathrm{~g} \cdot \mathrm{m}^{-2}$ and a cylinder diameter of $460 \mathrm{~mm}$ at $175 \mathrm{rpm}$ is

$$
\mathrm{p}_{\text {tot }}=17,4 \mathrm{~Pa}+45 \mathrm{~Pa}=62,4 \mathrm{~Pa} \text {. }
$$

This value should be regarded as a minimum estimation. It can be expected that the needed practical pressure value can be higher, about $100 \mathrm{~Pa}$ approx., but the verification must be made by experiment. This value is further used as the basis for an aerodynamic solution in section 3 . 


\subsubsection{Sketch of complex effect}

In the reality, all the above mentioned forces are acting together. The sheet is pushed away from the rotating cylindrical surface by the dominant centrifugal force so that its frontal surface is increasing and the pressing aerodanymic effect of the surrounding air, too. This effect is solvable as a mutual interaction of the fluid with the deforming elastic structure, only.

The result of the combined force effects from section 2.1 and 2.2 is the paper separating from the rotating surface, the free end fluttering, etc. The entire situation is variable in time; the separated paper end has a greater aerodynamic resistance comparing with the paper in contact with the surface. And more, during the paper passage through the machine, its free end length is shortening and the fluttering frequency is increasing. It should be to realize such additional contactless force effect which could press the free end of the paper to the rotating cylindrical surface in any position. As a suitable solution, the influence of free air flows onto the paper surface is coming into question.

\section{Aerodynamic pressing [7]}

The aim is to design and to solve several models, creating such a continuous force actuating of designed air flows, similar to the results of elasticity solutions as in the previous section 2.2. The load, necessary for paper sheet holding at the cylindrical surface, should be created in a contactless manner, using radial air flows. There is used the so-called wall effect when the air flow is well attached to the cylindrical surface. At the same time, the small volume of the primary flow takes more volume of the secondary air flow from the surroundings so that the needed effect is achieved relatively efficiently.

\subsection{Plane (2D) model}

The plane (2D) model is suitable for the given geometry, its creation is simple and the solution is quick. Two cases were solved, each for 5 inlets with distances of $10^{\circ}$ or $20^{\circ}$ along the cylinder periphery. Each case was solved with or without covers among the individual inlets. The force effect of individual flows and air consumption, too, is possible to change by changing the inlet gap width, inlet pressure, distance from the surface, number of inlets (nozzles), i.e. of various pitch (here of $10^{\circ}$ or $20^{\circ}$ ).

In the serial of figures 3.1-1 to 3.1-3, there are presented streamlines and isolines of both velocity and pressure, for the case with covers, a pitch of $10^{\circ}$. The same situation without covers is showed in figures 3.1-4 to 3.1-6.

In general, the set of wall nozzles creates appropriate set of air "cushions", which prevent contact of the treated paper sheet with machine structure.

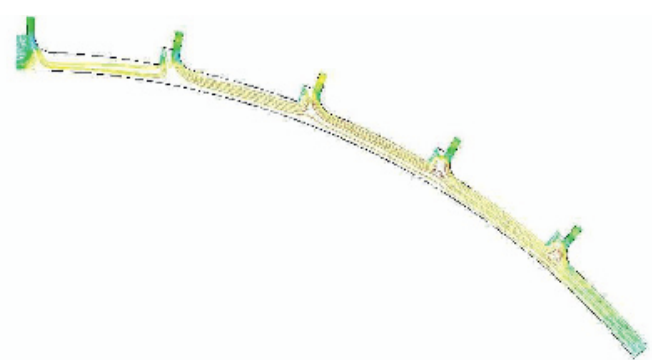

Figure 3.1-1. Streamlines - with covers.

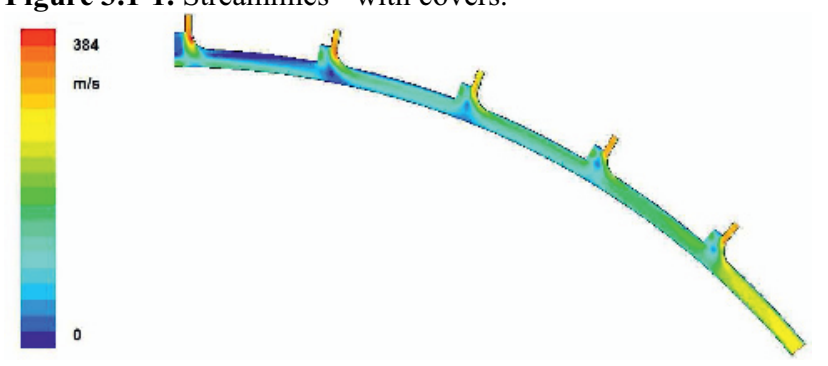

Figure 3.1-2. Velocity - with covers.

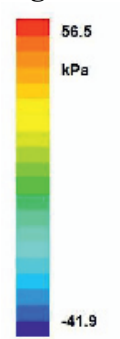

Figure 3.1-3. Pressure - with covers.

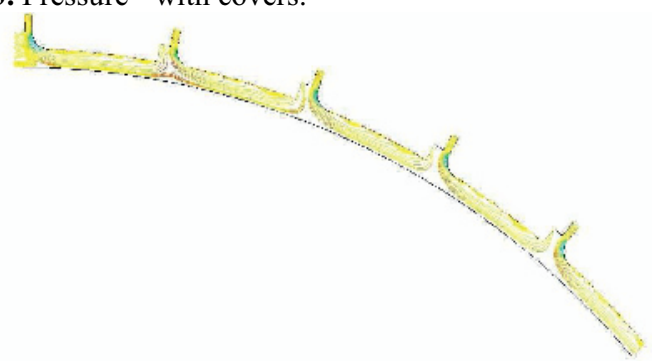

Figure 3.1-4. Streamlines - without covers.
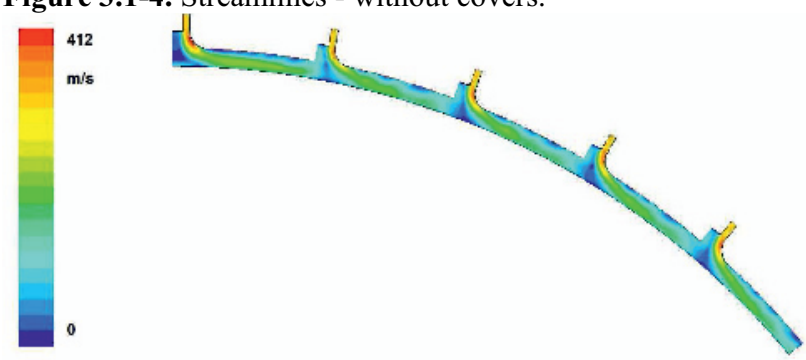

Figure 3.1-5. Velocity - without covers.

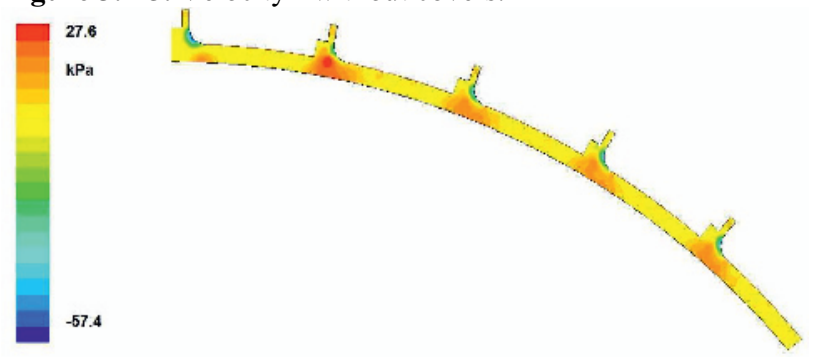

Figure 3.1-6. Pressure - without covers.

The common graph in figure 3.1-7 shows the pressure profiles of some solved cases. Of course, the profile for 4 
pitches of $10^{\circ}$ is shorter than that for $20^{\circ}$ on the same cylinder diameter.

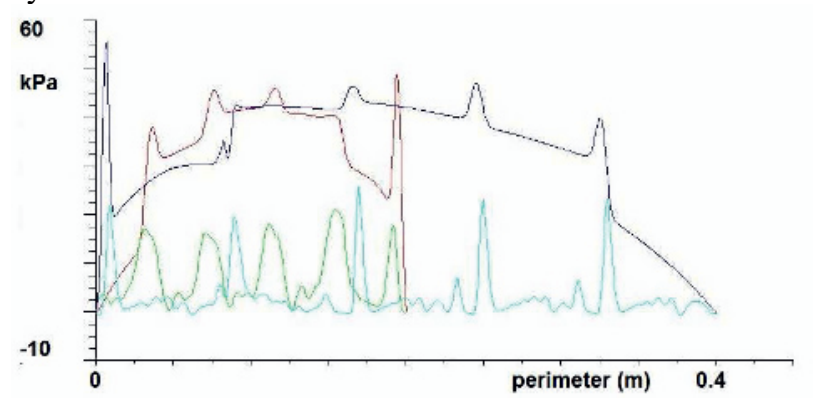

Figure 3.1-7. Pressure profile along the perimeter.

The covered perimeter between the inlets creates rather a continuous load with local tips in the areas of the inlets and with decreasing to the beginning and ending.

The uncovered perimeter creates rather isolated local picks in the areas of the inlets and with a very small continual load between the inlets.

\section{Preliminary results}

- By the wall effect, the radial streams are reliably bent into the peripheral direction in which moves the paper sheet.

- It is supposed that a stable and strong wall flow will have the effect of a cushion. Should such pressure effect of the flow could not take the paper sheet permanently on the cylindrical surface, the sheet will move closer to the inlets, where the aerodynamic force effect is stronger. In such a manner, some balanced sheet position is creating, we suppose that the full pressing of the sheet to the cylinder surface is not necessary.

- To get a higher reliability of the bend of flow, together with air consumption decreasing it should be possible to use narrower inlet gaps.

- The influence of covering between the inlets is essential: The cases with covers have a high average value of pressure, with small local tips in the areas of the inlets. For prospective maintenance it is a less suitable solution, the dismantling is necessary. On the other side, the cases without covers have a lower pressure value in general, with high picks in the areas of the inlets.

- A specified pressure value in the inlet creates a sufficient pressure effect on the cylinder surface. By iterative calculation it is possible to get a suitable pressure value which ensures both pressing effect and low consumption of compressing energy.

- To reduce the air consumption at the working frequency of $5 \mathrm{~Hz}$ (a time period of $200 \mathrm{~ms}$ ) it is possible to use a verified method of timing individual inlets, used on air jet looms until $20 \mathrm{~Hz}$ approx. The influence of operating delay between the electric signal for valve opening and full air flow is possible to determine by real measuring on machine (typical results are presented for instance in figure 4.1-9).

\subsection{Three-dimensional (3D) model}

A 3D model is necessary for the solution of mutual interactions of several streams after section 3.1, further for the description of boundary influences at the cylinder faces and in the middle symmetry plane and for the evaluation of flow uniformity or pressing uniformity along the whole length of the inlet gap. The inlet channel has two inlets, then with central symmetry plane. Then it is enough to solve one half of the model, only. At the same time, the periodic boundary condition is used - the flow field from the first gap is coming into the area of the second gap. To keep the condition of constant pressure along the whole inlet channel, it is necessary to keep the gap cross section considerably smaller than the inlet cross section. And more, it is necessary to get the pressure field similar to the result of the solution from section 2.2.

The cross section of the model is presented in figure 3.2$1-$ linked to the planar shape from section 3.1.
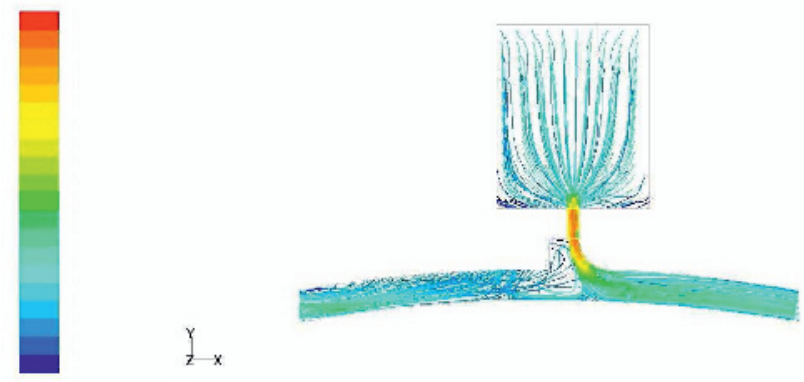

Figure 3.2-1. Streamlines from one wall nozzle into the cylinder periphery.

The air is flowing through the upper channel (perpendicular to the text plane). The following narrow gap is split into several parts, due to manufacturing and rigidity of the inlet structure. The pressing effect is divided into several parts, too.

At the gap outlet, the air flow is separating from (left) sharp outlet edge and is flowing along (right) rounded edge, so the whole flow is bending to the right. The vertical (radial) plane in the left from this outlet is the side inlet into the surroundings, which could be shut after operational conditions. The lower radius is the cylinder surface, where the sheet of paper should be hold along. Two cylindrical peripheral covers are situated concentrically in a small distance from the cylinder surface. They are opening as a peripheral outlet - it depends on the solved case. On the radial boundary planes between the cylinder and the cover it is defined a periodical boundary condition.

Constants of the solution:

- The distance of the cylinder surface from the gap outlet is constant, that of $20 \mathrm{~mm}$, the same as in the actual case.

- Air pressure in the inlet channel is $300 \mathrm{~Pa}$ (const.).

- The length of the modeled periodical part is of $20^{\circ}$ of the cylinder periphery.

- The defined constant rotation of the smooth cylinder surface $(-15,7 \mathrm{rad} / \mathrm{s})$, following the bend flow from the gap. 
- The peripheral cylindrical cover is divided into two parts, dismountable after the conditions of the solved case.

- The volume of the lengthwise gap is divided in several parts, blocked after the solved case.

- The side radial gap along the gap outlet is divided, too, and blocked after the solved case, too.

- The face gap between the cylinder and the peripheral cover is permanently shut. The boundary influences in the vicinity of terminal gaps in the peripheral direction are analogous to the results of the planar solution after section 3.1, not presented here.

In figures $3.2-2$ to $3.2-4$, there are presented some results of the simulation of the whole system of 7 long gap nozzles from figure 3.2-1, including a double-sided air inlet in common manifold.

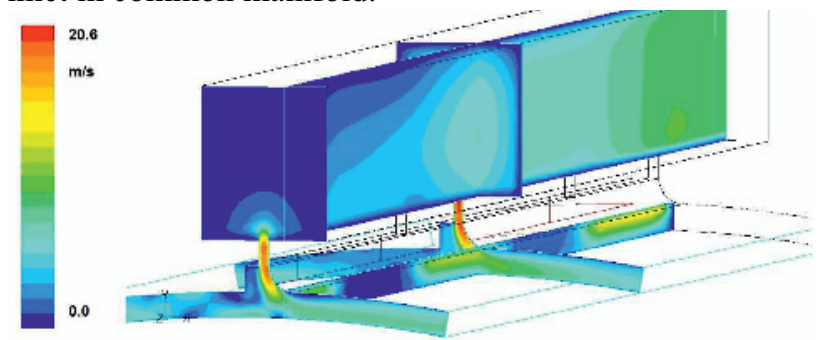

Figure 3.2-2. Possible air inlet for one gap - velocity field.

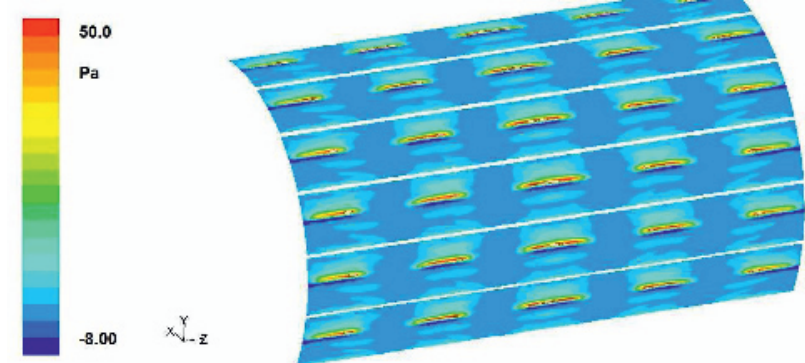

Figure 3.2-3. Traces of several gaps on the cylindrical surface.

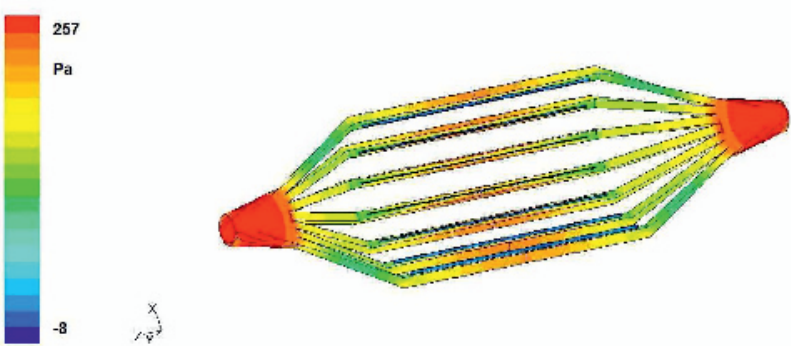

Figure 3.2-4. Global pressure field in inlets and gaps.

Assuming the fan efficiency of 0,5 approx. and the air density of $1,18 \mathrm{~kg} \cdot \mathrm{m}^{-3}$ for a pressure of $300 \mathrm{~Pa}$, the calculated power supply reaches of some Watts, only. For the gap pitch of $20^{\circ}$ it should be necessary to use 9 gaps approx., so the consumption of air and energy will be 18 times higher, together $54-113 \mathrm{~W}$. This value does not disturb the total energy consumption of the whole machine.

Conclusions:

- The designed cover shape with active nozzles (gaps) fulfils the needed effect: the contactless pressing of the paper sheet to the cylindrical surface. Even if it should be taken into account some flattering of the paper back end, the used stable air flows, arising from the lengthwise gaps, will be acting as an air cushion between the paper and the nozzles body.

- It is necessary to prevent the flow separation from the rounded outlet edge, then the cushion effect should be smaller. A suitable solution is given by another combination of air outlets into the surroundings, it should be to use narrower gaps, too. In such a way, the air consumption is decreasing, too.

- By a suitable arrangement of positions and dimensions of air outlets into the surroundings it is possible to reach an overpressure on the whole cylindrical surface. Some under pressure areas could help to the paper separation from the cylindrical surface and to the possible paper flattering, but such movements are very small.

- Some small irregularity of velocity or pressure field at the face of the cylinder relative to its centre is visible here. Probably, it is not any problem. The necessary pressing is changing in time and position, too, during the paper passage through the machine.

\section{Realization}

The above presented results of numerical flow simulations demonstrate the usability of designed contactless pneumatic pressing of the treated paper sheet to the cylinder surface. Unfortunately, regardless of such results, the idea was realized differently, simply as a tube with several crosswise drilled holes. Such kind of pneumatic pressing is relative simple and functional, but with a high noise level and high air consumption. And more, the pressing effect is spread in all directions, it means not only along the paper movement, but across and against it, too. On the other side, the design after section 3.2 is more complicated.

\subsection{Perforated tube [9]}

As an example of several models, there are presented results of layout as 1 hole of 1,5 $\mathrm{mm}$ and 2 holes of 1,0 $\mathrm{mm}$ with a distance of $20 \mathrm{~mm}$. All models are situated in the same volume, limited by the tube surface from up, where outlets are situated (an overpressure of $70 \mathrm{kPa}$ ), by cylinder surface from down and by a prism with a defined boundary condition "outlet" on the other sides. The tetrahedral mesh is refined during the solution in the areas of maximum velocity gradients, it is on the free flow surface and on the impact face, where could be the sources of maximum acoustical noise, too.

The results are presented as a velocity field in the vertical plane and as the pressure field, where at the suppressed scale, the details of the disturbed field are better visible and more the pressure field at the impact plane (turned of $90^{\circ}$ towards the front view). In white areas, the pressure value is lower than $-10 \mathrm{~Pa}$, it could cause small paper levitation, but it could be the problem of raw mesh, too. 

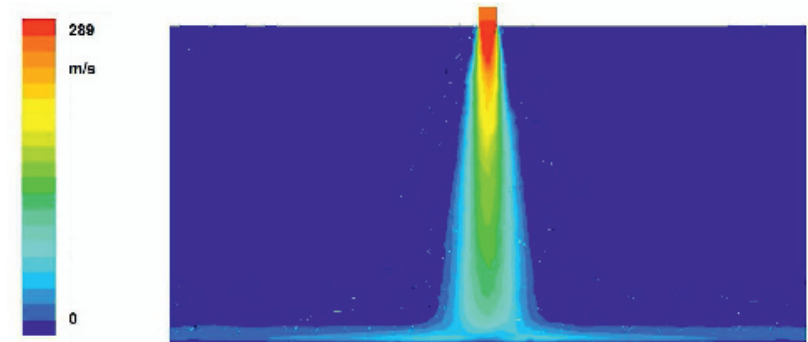

Figure 4.1-1. Velocity field, nozzle $1 \times 1,5 \mathrm{~mm}$
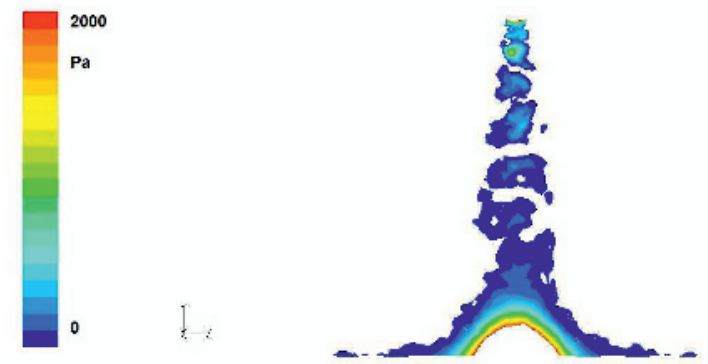

Figure 4.1-2. Pressure field, $1 \times 1,5 \mathrm{~mm}$ (suppressed)

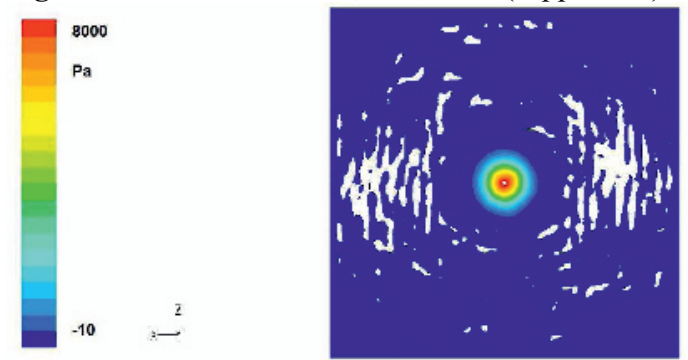

Figure 4.1-3. Pressure field at impact surface, $1 \times 1,5 \mathrm{~mm}$

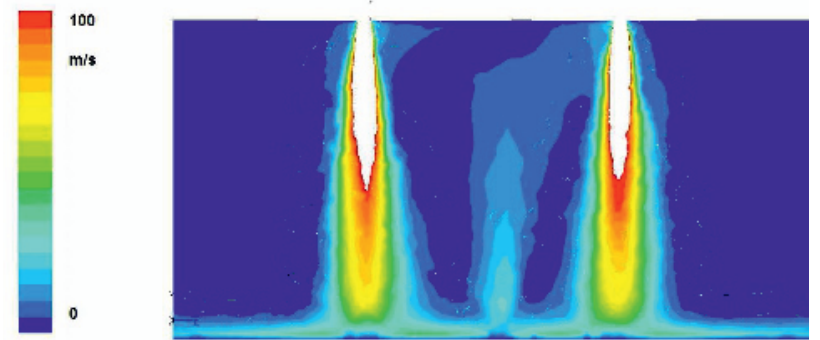

Figure 4.1-4. Velocity field, nozzles $2 \times 1,0 \mathrm{~mm}$

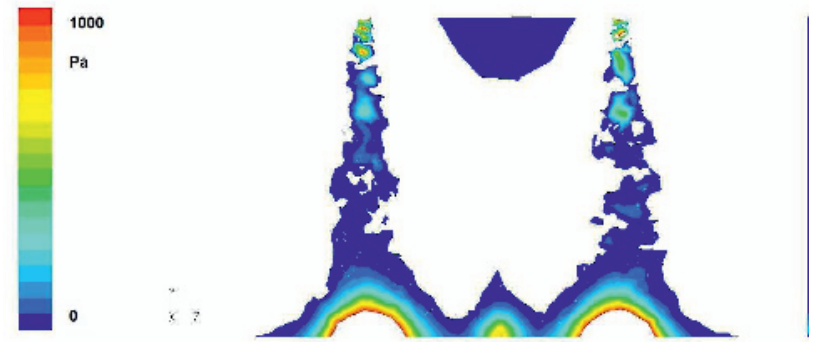

Figure 4.1-5. Pressure field, 2x1,0 mm (scale suppressed)

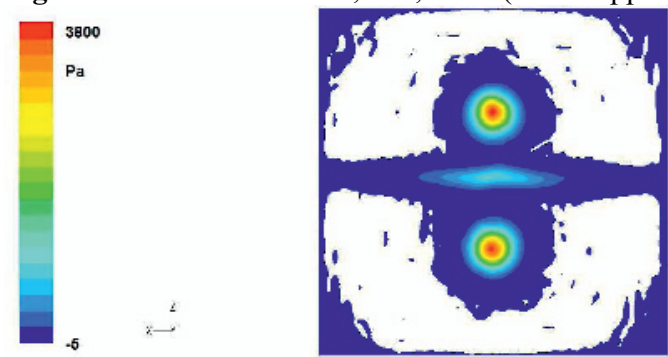

Figure 4.1-6. Pressure field at impact surface, $2 \times 1,0 \mathrm{~mm}$ (scale suppressed)

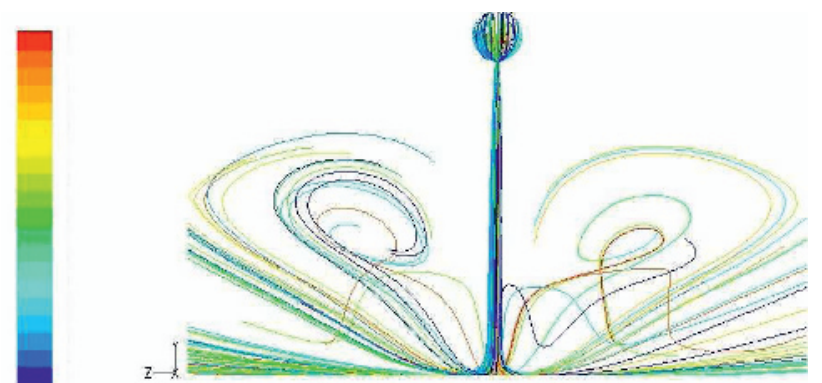

Figure 4.1-7. Streamlines in nozzle axis
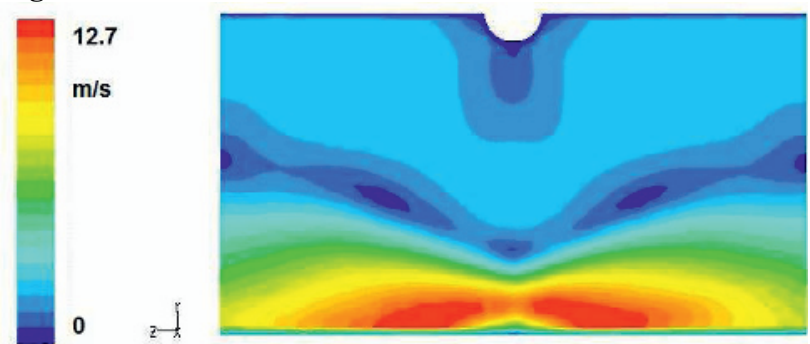

Figure 4.1-8. Velocity field between two adjacent nozzles

Maybe, the mutual interaction of two air flows has some influence, when some part of energy is nulled and is not affected on the impact plane.

From the received mass flows and total forces on the impact plane it is possible to evaluate the effectiveness of individual solved cases (various outlet diameters, their pitches, etc.).

\subsubsection{Aerodynamic noise decreasing}

1. The tube cross section (a diameter of $17 \mathrm{~mm}$ ) is much greater than the sum of individual outlets (14 times 1,5 $\mathrm{mm}$ ), it is enough to observe one outlet, only.

2. Instead of the given pressure of $160 \mathrm{kPa}$ (overcritical flow theoretically), it should be possible to use $70 \mathrm{kPa}$, only (just under critical flow theoretically). It is sure that the noise level of free flow is decreasing.

3. To check the quality and precision of outlet edges each small disturbing of the geometrically exact shape increases the aerodynamic noise and also decreases the range of the free flow. To reach a sufficient force effect on the impact plane it is necessary to increase air pressure. The noise level is increasing, too.

4. In general, the noise level is decreasing when one outlet is divided into several smaller outlets. The range of flow from smaller holes is decreasing, too, so it should be shortened the distance between the outlet and the impact plane.

5. Air jet looms use one-hole nozzles of $1,5 \mathrm{~mm}$ dia. or multi-hole nozzles 19 times $0,4 \mathrm{~mm}$ - they are not so noisy, even if the equivalent diameter is $1,7 \mathrm{~mm}$. It is possible to buy a similar multi-hole insert.

6. Use the gap nozzle, described above in section 3 and in [7]. The created air cushion prevents the paper contact 
with the tube surface. In general, it is possible to state that such a nozzle is silent, reliable, economic and simple for manufacturing. Of course, such design is more complicated relating to the drilled tube.

7. The noise level could be influenced by air flows interaction with the flattering paper sheet. The noise propagation in the surroundings can be passively suppressed by a suitable cover, but the manipulation during the machine maintenance could be complicated. A noise isolating effect can be reached by thick paper, carton, foam etc.

8. Air flow timing reduces the air consumption and also the time averaged noise level. The pressing effect is necessary along $25 \%$ of cylinder periphery approx., with a pause of about $25 \%$ (two paper sheets on the whole cylinder periphery). At the production output of 18000 papers per hour, the necessary switching frequency is $5 \mathrm{~Hz}$ with a pattern $50 \%$ open and $50 \%$ shut. Those times are long enough, no operational problems are expected.

After our experience the standard working frequency of air jet looms of $10-20 \mathrm{~Hz}$ is without problems. But it is necessary to fill directly the nozzles, without any large volume between the opened valve and the nozzle mouth, due to an arising long time delay.

From former record of air flow propagation on air jet loom we can read following typical timing for the working frequency of $10 \mathrm{~Hz}$ (working period of $100 \mathrm{~ms}$ ), see for instance [8].

The first trace presents the supply voltage $(50 \%$ on, $50 \%$ off), with an electronically suppressed induced pick just after the supply break - start at $0 \mathrm{~ms}$.

On the second trace there is the corresponding time course of the current, increasing due to real inductance and resistance of the coil - the beginning of valve plug movement at $28 \mathrm{~ms}$, the beginning of current decreasing at $50 \mathrm{~ms}$, identically with supply voltage interruption.

The third trace shows the time point when the valve is fully open - at $39 \mathrm{~ms}$. The impact of the valve plug is recorded by accelerometer. and the last one shows the gradually increasing dynamic pressure at the nozzle outlet.

The fourth trace presents the gradually increasing dynamic pressure of the air flow at the nozzle outlet - the $95 \%$ of maximum value reached at $44 \mathrm{~ms}$.

Result: In examinated configuration, the total delay reaches up of $44 \%$ of the working period of the machine. This time is the physical constant of the machine, with increased machine operation it presents higher percentage of the working period. It should be necessary to make some technical measures for its shortening.

\subsection{Fans [10]}

Another solution of contactless pressing uses air flow from axial fan(s), directed into the area of paper separation from the cylinder surface. Such a solution is used for the reason that the fans are available, used on the machine for another purpose. The problem could be a low pressure of axial fans in general. The next serial figure 4.2-1 to figure 4.2-4 shows the velocity and pressure field in two radial planes - in the fan axis and between two fans (pressed paper is situated down).

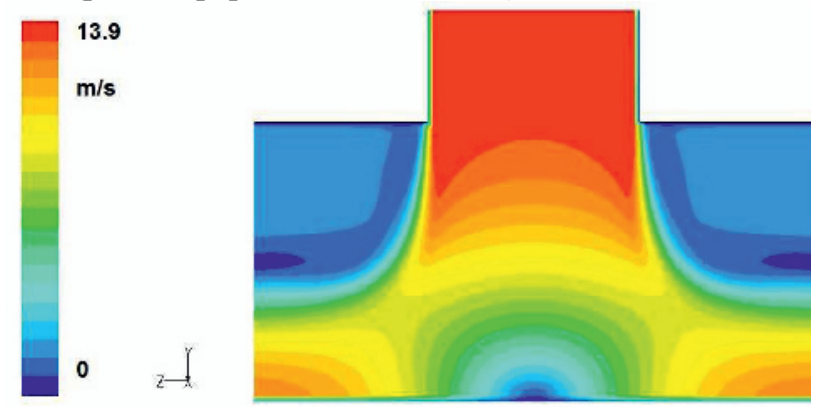

Figure 4.2-1. Velocity field in fan axis

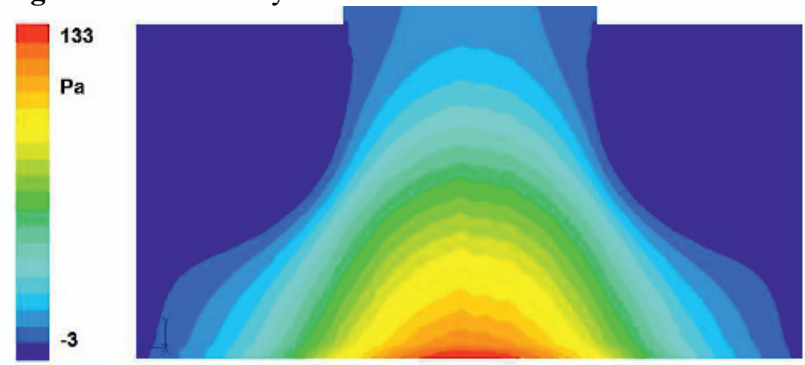

Figure 4.2-2. Pressure field in fan axis

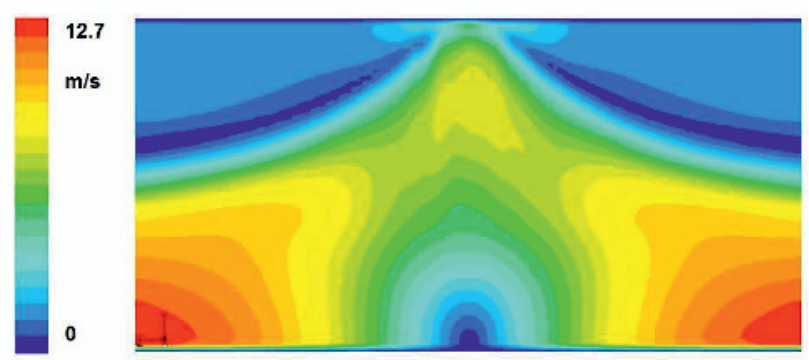

Figure 4.2-3. Velocity field between two fans

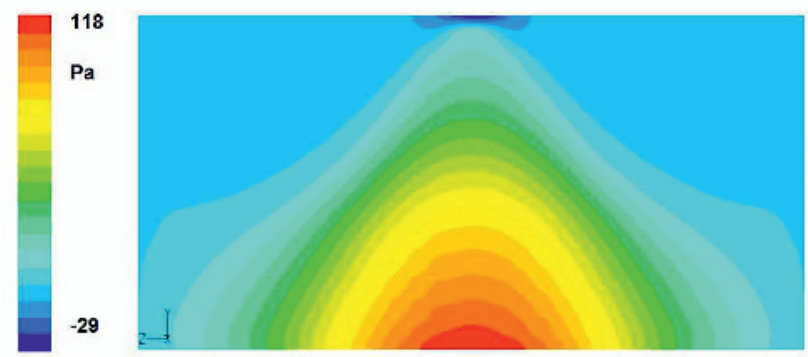

Figure 4.2-4. Pressure field between two fans

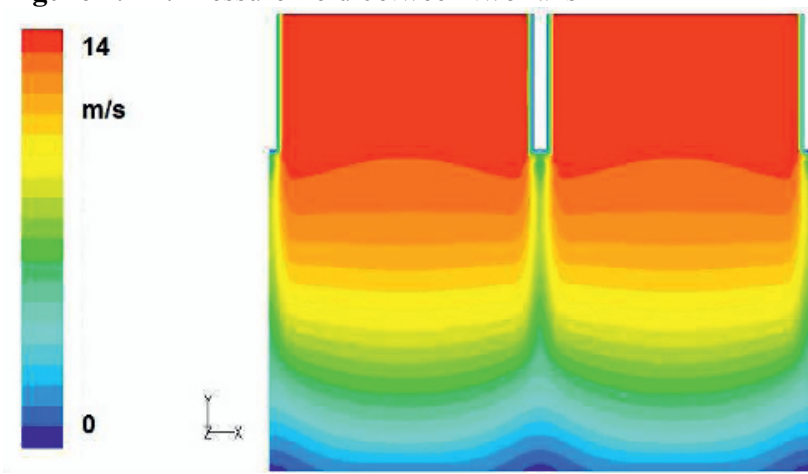

Figure 4.2-5. Velocity field in axial plane (2 fans)

The flow field description is completed by figure 4.2-5 velocity field in the axial plane (two adjoining fans) and figure 4.2-6 - pressure field on the impact plane. Here can 
be evaluated the total force effect on the treated paper sheet.

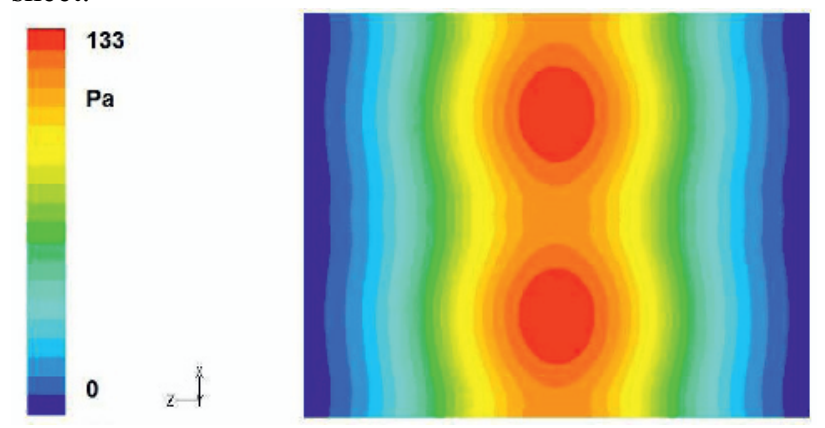

Figure 4.2-6. Pressure field at impact plane (2 fans)

\section{Conclusions}

The article presents the contactless "static" pressing of the paper sheet to the surface of the rotating cylinder, using gap nozzles with the so-called wall effect. Such flows are in general stable, with good directional stability and a relative low of both air consumption and noise level, too.

Using an alternative simple perforated tube leads to a high air consumption and high noise level.

A numerical simulation of the effect of air flows from the gap nozzles on the opposite impact plane results from the previous flow modelling around the various rotating cylinders $[1,2,3,4]$, completed by the results of elasticity calculations [7] and in section 2.2.1. At first, the simple planar (2D) model of air flow is solved, which indicates the possibility of contactless pressing of the treated paper sheet to the cylindrical surface. The next periodical spatial (3D) model describes the mutual interactions of individual flows and with the surroundings, too. Typical results are presented in section 3.1 and 3.2.

The results of elasticity calculations show that the maximum pressing should be at the end of the paper sheet, which is moving with a velocity of 3,0 to $4,2 \mathrm{~m}^{-} \mathrm{s}^{-1}$ relative towards the fixed nozzles. It should be taking into account that the end edge of the paper sheet will be standing away from the cylinder surface. The designed equipment should provide the acceptable value of such taking away (the actual design supposes not more than 30 $\mathrm{mm})$.

For the present, this first solution does not take into account the natural fluttering of the free end of the paper sheet. During the paper passage through the machine the fluttering frequency of the shortening free end of the paper is increasing and the amplitude is decreasing.

The reduction of air consumption by a timing of air inlets (for instance as the system of nozzles for air jet looms) is possible, when valves will be placed to the nozzle mouths as close as possible, to suppress the transporting delay at relative short times of working cycles.
There are presented two kinds of a solution - freely placed gap nozzles have a smaller pressing effect, an added cover between the nozzles suppresses the air escape into the surroundings and the pressing effect is increased. But it is necessary to let some free connection between the inner volume and the surroundings.

The presented "static" solution supposes that such pneumatic pressing of the paper sheet to the cylinder surface will ensure the paper position in some range relative to the cylinder surface. Really, many next factors are influencing, therefore, the paper position will not be stable but fluttering. For instance, the gap of $30 \mathrm{~mm}$ between the surfaces of the pressing and feeding cylinders in the area of paper transferring, designed nozzles are situated in some pitches and distances, possible air layer between the cylinder surface and the paper during the rotation, time-dependent length of the paper end and its fluttering with variable frequency, etc. The goal of the designed pneumatic pressing is to catch that radial movement in suitable limits.

Another pressing system uses standard axial fans. Both systems should be verified by real trials with an evaluation of reliability, economy, noise level and force effects.

\section{References}

1 K. Adámek, J. Pelant.: Aerodynamika mykacího válce, report No. PRO 400/2003, VÚTS Liberec, (2003), (in Czech)

2 K. Adámek, J. Pelant.: Luftströmung entlang des rotierenden Kardenbelags, Chemnitzer Textilmaschinen Tagung, TU Chemnitz, (2003), (in German)

3 J. Pelant, K. Adámek.: Flow around moving surfaces, ECCOMAS 2004, Univ. of Jyväskyla, (2004)

4 K. Adámek, J. Žák.: Luftströmung in einer Offsetdruckmaschine / Proudění vzduchu v ofsetovém stroji, report No. PRO-440/2005, VÚTS Liberec, (2005), (in German, in Czech)

5 K. Adámek.: Vliv proudění za rotace na užitné vlastnosti výrobku, report No. VAM 598/2011 (Pegas Znojmo), VÚTS Liberec, 2011, (in Czech)

6 J. Kolář, K. Adámek: Influence of the induced airflow on calendering, EFM 2011, TU Liberec (Jičín), (2011)

7 K. Adámek, J. Žák, P. Šidlof, P. Kavan: Durchgang des Bogens in der Offset-Druckmaschine / Průchod archu $\mathrm{v}$ ofsetovém stroji, report No. PRO-456/2006, VÚTS Liberec (2006), (in German, in Czech)

8 K. Adámek: Applied fluid mechanics - in Czech. Lectures for Fac. of Machinery at TU in Liberec (www.tul.cz/kez), (2007)

9 K. Adámek, J. Kolář: Hlučnost pneumatického př́tlaku studie řešitelnosti, report No. PRO-540/2009, VÚTS Liberec, (2009), (in Czech)

10 K. Adámek: Př́tlak archu papíru, report No. VAM-24/2010, VÚTS Liberec, (2010), (in Czech) 